\title{
Different Depression Treatment Recommendations and Adherence for Spanish- and English-Speaking Patients
}

\author{
Jacquelyn Stephenson, PhD, MSW, Brian Distelberg, $\mathrm{PhD}, \mathrm{MA}$, \\ Kelly R. Morton, PhD, MA, Larry Ortiz, PhD, MSW, and \\ Susanne B. Montgomery, PhD, MPH, MS
}

Introduction: National guidelines recommend primary care providers (PCPs) screen patients for depression with a standardized tool and address positive screenings. However, depression prevalence is lower in Latinos (8\% to 15\%, with Spanish speakers at $8 \%$ ) than non-Latino whites (22\%). As a result of these prevalence differences, PCPs may use ethnicity and language of the patient to determine depression screening behaviors. This study examined standard of care (SoC) depression treatment recommendations by ethnicity and language for patients who screened positive for major depression during a medical visit.

Methods: 275 patients scored $\geq 10$ on the Patient Health Questionnaire-9 screening; a chart review assessed treatment referrals, followed by semistructured interviews with 18 patients and 7 PCPs regarding depression treatment behaviors.

Results: 138 patients (50\%) received SoC treatment recommendations. After controlling for age, gender, and race, a binary logistic regression was performed to determine language and ethnicity effects on SoC depression treatment recommendations (psychotherapy/pharmacotherapy vs other). Spanishspeaking Latinos were $\mathbf{7 2} \%$ less likely to receive SoC recommendations than English speakers (odds ratio $[\mathrm{OR}]=0.39)$. Interviews with Spanish-speaking patients confirmed that negative perceptions about medications, patient noncompliance, and a shortage of bilingual behavioral health providers within the clinic impacted SoC recommendations and uptake.

Conclusions: Spanish-speaking Latinos did not receive or follow through with SoC recommendations as often as English speakers regardless of ethnicity. Future studies should explore reasons why language is a barrier to SoC recommendations for Latinos and explore culturally and linguistically sensitive methods to effectively treat Spanish speakers for depression during a medical visit. (J Am Board Fam Med 2019;32:904-912.)

Keywords: Behavioral Medicine, Clinical Decision-Making, Cross-Cultural Comparison, Depression, Hispanic Americans, Logistic Models, Medicaid, Mental Health, Patient Care Team, Patient Compliance, Patient Health Questionnaire, Primary Health Care, Psychotherapy, Qualitative Research, Referral and Consultation, Standard of Care

Up to $70 \%$ of primary care visits include psychosocial and mental health concerns ${ }^{1}$ and approximately $40 \%$ of patients with depression symptoms

This article was externally peer reviewed.

Submitted 2 November 2018; revised 11 June 2019; accepted 21 June 2019.

From Inland Empire Health Plan, Rancho Cucamonga, CA (JS); Behavioral Medicine Center, Loma Linda University, Redlands, CA (BD); School of Behavioral Health, Department of Counseling and Family Sciences, Loma Linda University, Loma Linda, CA (BD); School of Behavioral Health Loma Linda University, Loma Linda, CA (KRM); School of Behavioral Health, Department of Psychology, Loma Linda University, Loma Linda, CA (KRM); School of Medicine, Department of Family Medicine, Loma Linda University, Loma are first seen at a community health center. ${ }^{2}$ The Health Resources and Services Administration (HRSA) $)^{3}$ defines federally qualified health centers (FQHCs) as "community-based health care provid-

Linda, CA (KRM); Loma Linda University School of Behavioral Health, Social Work and Social Ecology, Loma Linda, CA (LO); School of Behavioral Health, Social Work and Social Ecology, Loma Linda University, Loma Linda, CA (SBM);

Behavioral Health Institute, Redlands, CA (SBM).

Funding: none.

Conflict of interest: none declared.

Corresponding author: Jacquelyn Stephenson, $\mathrm{PhD}, \mathrm{MSW}$ Loma Linda University School of Behavioral Health, 1686 Barton Road, Suite 100, Redlands, CA 92373 (E-mail: jstephenson@llu.edu). 
ers that receive funds from the HRSA Health Center Program to provide primary care services in underserved areas." FQHCs may be community health centers and must meet a stringent set of requirements for continuation of funding, including annual reporting on a set of clinical quality measures such as the rate of annual depression screening with a standardized assessment tool, and, if positive the documentation of a follow-up plan. ${ }^{4}$ FQHCs often follow guidelines from the United States Department of Health and Human Services, Agency for Healthcare Research and Quality to meet these clinical quality metrics; however, these guidelines do not specifically address potential culturally sensitive concerns in screening and followup for mental health diagnoses. The recommended evidence-based guideline ${ }^{5}$ for depression screening, diagnosis, treatment, and followup from the Institute for Clinical Systems Improvement (ICSI) does include cultural considerations (religion, health care beliefs, effective communication, etc.) when implementing depression-screening and follow-up processes that are of importance for Latino patient populations.

The United States Census Bureau reports that Latinos comprise approximately $17 \%$ (54 million) of the total US population. ${ }^{6}$ However, Latinos are poorly represented in large clinical studies of depression, usually due to language exclusion criteria. ${ }^{7}$ Approximately $55 \%$ of the adult Latino population reports Limited English Proficiency in the United States ${ }^{8}$; therefore, significant linguistic barriers exist for high-quality screening, diagnosis, treatment, and management of depression in this population. Additional barriers to depression treatment uptake faced by Spanish-speaking Latinos include medication and psychotherapy costs, transportation, low English literacy levels, lack of Spanish-speaking therapists, distrust of medical providers, as well as a loss of social support to seek diagnosis and treatment due to immigration and acculturation issues. ${ }^{8-12}$ Being consistently screened for depression symptoms and receiving standard of care (psychotherapy/ pharmacotherapy) treatment recommendations when appropriate during a medical visit are necessary components of high-quality care that may vary by cultural group due to these other contextual concerns.

The present mixed-methods study participants are a subset of patients from a larger study con- ducted by the authors. ${ }^{13}$ The larger study (2017) analyzed administration and screening outcomes of a standardized, brief depression-screening tool, the Patient Health Questionnaire-2 (PHQ-2) in an FQHC setting with integrated behavioral health. Of the 10,285 adult patients seen during a medical visit in 2014 and/or 2015, only 46\% (4745) were screened for depression with the PHQ-2. Results found higher screening rates for Latinos, yet Spanish speakers were $31 \%$ less likely to screen positive on the PHQ-2 compared with English speakers regardless of ethnicity. The larger study helped to inform the design of the present study, whose purpose is to add valuable information to the few culturally and linguistically inclusive studies examining depression treatment recommendations for Latino populations scoring 10 or greater on the PHQ-9 and in need of a primary care provider (PCP) treatment recommendation. Specifically, we examine here whether language and/or ethnicity impact PCP's standard of care recommendations for treatment of depression. The results of this study can inform physicians, health care organizations, and mental health providers of the need to consider cultural and linguistic factors affecting depression screening and depression treatment in $\mathrm{La}-$ tinos.

\section{Methods \\ Participants}

\section{Quantitative Participants}

A mixed-methods design was used to explore standard of care depression treatment recommendations for patients screened for depression at an FQHC that treats patients insured primarily with a Medicaid Health Maintenance Organization health plan. First, a chart review was conducted for 275 patients who screened positive for depression during a medical visit to examine the treatment recommendations prescribed by their PCP. This was approved as an expedited archival chart audit by the institutional review board to obtain access to electronic medical records for eligible FQHC patients. The first author conducted an electronic chart review and included patients who screened $\geq 10$ on the PHQ-9 during any medical visit in 2014 to 2015, reported their ethnicity as either Latino or Non-Latino, reported their preferred language as either English or Spanish. Chart audits of these 275 patients were completed to ascertain the PCP's 
treatment recommendations in the Plan section of the visit note linked to depression diagnosis.

\section{Qualitative Participants}

Qualitative interviews were conducted, through purposive sampling assuring triangulation, to explore standard of care depression treatment recommendations through an ethnic-linguistic analysis for 18 patient participants and 7 PCP participants. Six English-speaking non-Latinos ( 3 males and 3 females), 6 English-speaking Latinos (1 male and 5 females), 6 Spanish-speaking Latinos (1 male and 5 females), and 7 PCP's (2 males and 5 females) participated in the interviews. Patients were recruited with flyers and scripted invitations by nursing staff after clinic visits. Potential participants contacted the research team and were subsequently consented and interviewed within 2 months of recruitment, allowing the patients time for treatment recommendation uptake. Participants were offered a $\$ 5$ gift card for their time.

\section{Procedures}

\section{Quantitative Procedures}

Data were deidentified and stored in a passwordprotected spreadsheet on a secure server. Data collected included gender (male or female), ethnicity (Latino or non-Latino), race (white or not white), language preference (English or Spanish), PHQ-9 scores, and PCP depression treatment recommendations (pharmacotherapy medication prescribed, in-house or outpatient psychotherapy referral, or both). Quantitative data were analyzed using SPSS, version 23.0 (IBM, Armonk, $\mathrm{NY}$ ) to conduct a binary logistic regression analysis.

\section{Qualitative Procedures}

We used the qualitative research method by Charmaz and Belgrave (2012). ${ }^{14}$ The primary author received permission to modify the interview guide of Horevitz, Organista, and Arean ${ }^{18}$ to align the questions with the FQHC's integrated setting. The FQHC utilizes Integrated Behavioral Health Specialists to provide same-day behavioral health triage and followup in a medical setting to reduce stigma of mental health treatment and to integrate mental health care within the primary care team for more effective and high-quality patient care experiences.
Qualitative interviews lasting 30 to 40 minutes were conducted in English and Spanish by a bilingual, qualitatively trained interviewer using a semistructured key informant guide to explore patient responses to the PCP recommendations, follow-up engagement of the treatment recommendations, and reasons for their treatment recommendation responses.

Member checking is a technique used to improve internal validity; the process provides a forum to check the viability of the themes created by researchers, correct results, and assess the accuracy of the data reported. For member checking, we conducted validation focus groups with 4 participants from each patient subgroup and 9 providers. Patient focus groups included both interview participants and new eligible patients. Provider focus groups also included both interview participants and new eligible PCPs.

Both interviews and focus groups were audio recorded and transcribed verbatim. Spanish-language transcripts were translated by a certified translator and back translated to ensure language equivalency. Inductive and deductive methods were used to analyze the interviews. A literature review provided deductive themes to form hypotheses while inductive methods allowed themes to emerge in the development of a codebook. The interview data were coded with Qualitative Data Analysis Miner, Version 5 (Montreal, Canada) using grounded theory. All transcripts were coded by 2 coders and agreement/disagreement was explored with constant comparison methods, reaching 94\% congruence. The codebook reflected both theoretical and emergent themes from interviews. The authors explored how a positive, negative, or neutral perception of psychotherapy and pharmacotherapy may have differed between and among groups.

\section{Measures \\ Patient Health Questionnaire-9}

The Patient Health Questionnaire-9 (PHQ-9) is an instrument based on DSM-V diagnostic criteria for depression, designed for use in primary care settings ${ }^{15}$ that includes 2 items from the short form (PHQ-2) and 7 additional items rated on a 4-point Likert scale (0-not at all to 3-nearly every day). A PHQ-9 cut-score of 10 has an $88 \%$ sensitivity and $88 \%$ specificity for the diagnosis 
of major depressive disorder. ${ }^{16}$ According to Institute for Clinical Systems Improvement, "the PHQ-9 has been validated as a tool for both detecting and monitoring depression in primary care settings." The PHQ-9 has been validated for use in primary care settings and is comparable when tested with Latino and non-Latino patient groups. ${ }^{17}$

\section{Major Depressive Disorder Treatment Recommendations}

To identify the PCP follow-up treatment recommendations and the uptake of the recommendations by the patient, the electronic medical record was queried for behavioral health follow-up appointments in the FQHC and prescriptions for antidepressants in the medication list. The Institute for Clinical Systems Improvement's Health Care Guideline: Depression in Primary Care ${ }^{5}$ (referred to as the FQHC Standard of Care throughout this manuscript) specifies that patients with a score of $\geq 10$ on the PHQ-9 should receive treatment recommendations of combined psychotherapy and pharmacotherapy. The study clinic site has a written guideline based on ICSI recommendations in collaboration with an Integrated Behavioral Health Specialist available on-site: provide same-day behavioral health consults, diagnosis, and treatment recommendations during the medical visit. Chart audits of the Plan section of the PCP visit note (for the visit when depression was diagnosed) was coded as YES standard of care recommended/prescribed (psychotherapy/pharmacotherapy) versus NO standard of care was not recommended/prescribed (psychotherapy only, pharmacotherapy only, education/breathing exercises/physical exercise, or no recommendation).

Although the study site uses ICSI as a basis for their internal depression screening guideline, the ICSI guideline differs from many other guidelines in that combined treatment is recommended for all patients, even those with only mild-to-moderate depression. The guideline actually indicates that the marginal gain is relatively small and that in many patients, initial monotherapy with psychotherapy or pharmacotherapy may not only be what is feasible but also appropriate; thus, the authors also conducted a sensitivity analysis by coding those that received either psychotherapy or pharmacotherapy recommendations as YES.
Table 1. Demographic Characteristics for Patients with $P H Q \geq 10$ between 2014 and 2015

\begin{tabular}{lc}
\hline Variables & $\mathrm{n}(\%)$ \\
\hline $\mathrm{n}$ & 275 \\
Mean Age [SD], years & $44.92[13.61]$ \\
Mean PHQ-9 [SD], score & $18.10[4.32]$ \\
Gender & \\
Female & $202(73.5)$ \\
Male & $73(26.5)$ \\
Race & \\
White & $171(62.2)$ \\
Black/African American & $43(15.6)$ \\
Asian & $4(1.5)$ \\
American Indian/Alaskan & $5(1.8)$ \\
$\quad$ Native & $1(0.4)$ \\
Native Hawaiian/Pacific & \\
$\quad$ Islander & $4(1.5)$ \\
More than one race & $47(17.1)$ \\
Unknown/not reported & \\
Patient ethnicity & $135(49.1)$ \\
Latino & $140(50.9)$ \\
Not Latino & \\
Patient's preferred language & $248(90.2)$ \\
English & $27(9.8)$ \\
Spanish &
\end{tabular}

SD, standard deviation; PHQ-9, Patient Health Questionnaire-9.

\section{Results}

\section{Chart Review}

Table 1 provides demographic characteristics for 275 patients included in the study. Participants' ages ranged from 18 to 85 years $(\mu=44.92$; $\mathrm{SD}=$ 13.61). The majority of patients were female (73.5\%), white (62.2\%), English speakers (90.2\%), and about half of the participants were Latino (49.1\%). Mean PHQ-9 score was 18.10 (SD = 4.32), indicating moderate to severe depression. About half of patients received combined psychotherapy/pharmacotherapy treatment recommendations $(48.4 \%)$, whereas $44 \%$ of patients received monotherapy recommendations (96 received psychotherapy only and 27 received pharmacotherapy only). The remaining $7 \%$ of patients received PCP psychoeducation-based interventions, such as physical activity counseling, breathing exercises, or depression education.

A binary logistic regression analysis was conducted to assess the association between language and ethnicity on psychotherapy/pharmacotherapy depression treatment recommendation after controlling for age, gender, and race in the first 
Table 2. Logistic Regression of Demographic Characteristics Predicting Combined Depression Treatment Recommendations

\begin{tabular}{|c|c|c|c|c|c|c|c|c|c|}
\hline \multirow[b]{3}{*}{ Variable } & \multicolumn{3}{|c|}{ Model 1} & \multicolumn{3}{|c|}{ Model 2} & \multicolumn{3}{|c|}{ Model 3} \\
\hline & \multicolumn{3}{|c|}{$P=.27$} & \multicolumn{3}{|c|}{$P=.29$} & \multicolumn{3}{|c|}{$P=.04^{*}$} \\
\hline & OR & $P$ & $95 \% \mathrm{CI}$ & OR & $P$ & $95 \% \mathrm{CI}$ & OR & $P$ & $95 \% \mathrm{CI}$ \\
\hline Constant & 1.96 & .13 & & 1.76 & .22 & & 4.24 & .02 & \\
\hline Age & 0.98 & .07 & $0.97,1.00$ & 0.98 & .06 & $0.97,1.00$ & 0.98 & .03 & $0.96,1.00$ \\
\hline Male gender & 0.88 & .63 & $0.51,1.51$ & 0.88 & .64 & $0.51,1.52$ & 0.92 & .76 & $0.53,1.60$ \\
\hline White race & 1.18 & .51 & $0.72,1.95$ & 1.20 & .48 & $0.73,1.98$ & 1.20 & .47 & $0.73,2.00$ \\
\hline Latino ethnicity & & & & 1.30 & .29 & $0.80,2.10$ & 1.58 & .08 & $0.94,2.65$ \\
\hline \multirow[t]{2}{*}{ Spanish language } & & & & & & & 0.39 & .04 & $0.16,0.95$ \\
\hline & \multicolumn{3}{|c|}{ Classification $=56.7 \%$} & \multicolumn{3}{|c|}{ Classification $=54.2 \%$} & \multicolumn{3}{|c|}{ Classification $=54.2 \%$} \\
\hline
\end{tabular}

${ }^{*} P<.05$.

$\mathrm{OR}$, odds ratio; $\mathrm{CI}$, confidence interval.

block. The model was not a significant fit to the data $\chi^{2}(3,275)=3.92 ; P=.27$. The inclusion of ethnicity in block 2 was also not a significant fit to the data $\left[\chi^{2}(1,275)=1.14 ; P=.29\right]$. However, the inclusion of language in the third block was significant $\left[\chi^{2}(1,275)=4.45 ; P=.04\right]$. The final model correctly classified $54.2 \%$ of cases (Table 2).

Evaluation of odds ratios and corresponding confidence intervals (95\%) within the full model were used to examine the strength of the individual predictors. Criterion for significance was set at $P<.05$. Age and language were the only significant predictors of psychotherapy/pharmacotherapy depression treatment recommendations following a depression score of 10 or greater on the PHQ-9. Age was estimated to have an odds ratio $[\mathrm{OR}]=0.98$ and English language was estimated at $\mathrm{OR}=0.39(P<.05)$. These results indicate that older, and, Spanish-speaking (all were Latino in this sample) Latinos were less likely to receive a combined psychotherapy/pharmacotherapy depression treatment recommendation from their PCP after a positive depression screening (PHQ-9 $\geq 10$ ).

A sensitivity analysis was also conducted to assess the association of language and ethnicity on psychotherapy or pharmacotherapy depression treatment as standard of care recommendations after controlling for age, gender, and race in the first block. The model was not a significant fit to the data $\chi^{2}(3,275)=3.87$; $P=.41$. The inclusion of ethnicity in block 2 was a significant fit to the data $\left[\chi^{2}(1,275)=4.23 ; P=.04\right]$. However, the inclusion of language in the third block was not significant $\left[\chi^{2}(1,275)=1.28 ; P=.26\right]$. The second model correctly classified $90.9 \%$ of cases (Table 3).

Evaluation of odds ratios and corresponding confidence intervals (95\%) within the full model were used to examine the strength of the individual predictors. Criterion for significance was set at $P<$ .05 . Ethnicity was the only significant predictor of monotherapy (psychotherapy or pharmacotherapy) depression treatment recommendations following a depression score of 10 or greater on the PHQ-9. Ethnicity was estimated to have an $\mathrm{OR}=0.40, P=$ .04. These results indicate that Latino patients were less likely to receive a psychotherapy or pharmacotherapy depression treatment recommendation from their PCP after a positive depression screening (PHQ-9 $\geq 10$ ) regardless of language spoken.

\section{Qualitative Interviews}

Inductive and deductive qualitative analyses expanded on quantitative results by exploring themes related to how ethnicity and language may influence standard of care depression treatment recommendations and uptake. Table 4 summarizes qualitative findings by patient interview group into 3 primary themes 1): perception of depression treatment recommendations (including medications, psychotherapy, and a combined psychotherapy/pharmacotherapy recommendation), 2) receipt of depression treatment recommendations, and 3) barriers impacting uptake of treatment recommendations. 
Table 3. Logistic Regression of Demographic Characteristics Predicting Depression Treatment RecommendationsSensitivity Analysis Including Monotherapy

\begin{tabular}{|c|c|c|c|c|c|c|c|c|c|}
\hline \multirow[b]{3}{*}{ Variable } & \multicolumn{3}{|c|}{ Model 1} & \multicolumn{3}{|c|}{ Model 2} & \multicolumn{3}{|c|}{ Model 3} \\
\hline & \multicolumn{3}{|c|}{$P=.39$} & \multicolumn{3}{|c|}{$P=.04^{*}$} & \multicolumn{3}{|c|}{$P=.26$} \\
\hline & OR & $P$ & $95 \% \mathrm{CI}$ & OR & $P$ & $95 \% \mathrm{CI}$ & OR & $P$ & $95 \% \mathrm{CI}$ \\
\hline Constant & 20.97 & .001 & & 41.02 & .001 & & 47.47 & .001 & \\
\hline Age & 0.98 & .16 & $0.95,1.01$ & 0.98 & .12 & $0.95,1.01$ & 0.98 & .08 & $0.94,1.00$ \\
\hline Male gender & 1.57 & .33 & $0.64,3.86$ & 1.60 & .31 & $0.65,3.96$ & 1.61 & .31 & $0.65,3.99$ \\
\hline White race & 0.98 & .96 & $0.40,2.38$ & 1.00 & .99 & $0.41,2.46$ & 0.97 & .94 & $0.39,2.39$ \\
\hline Latino ethnicity & & & & 0.40 & $.05^{*}$ & $0.16,1.00$ & 0.35 & .03 & $0.14,0.89$ \\
\hline \multirow[t]{2}{*}{ Spanish language } & & & & & & & 2.29 & .30 & $0.48,10.91$ \\
\hline & \multicolumn{3}{|c|}{ Classification $=90.9 \%$} & \multicolumn{3}{|c|}{ Classification $=90.9 \%$} & \multicolumn{3}{|c|}{ Classification $=90.9 \%$} \\
\hline
\end{tabular}

${ }^{*} P<.05$.

$\mathrm{OR}$, odds ratio; CI, confidence interval.

Theme 1: Perception of Depression Treatment Recommendations

The perception of depression treatment recommendations varied by modality. Ethnicity played a role in perceptions of both pharmacotherapy and psychotherapy. Most Latinos reported negative perceptions toward depression medication treatment recommendations whereas English-speaking non-Latinos expressed positive benefits to depression medications. While most Latinos supported psychotherapy for depression treatment, surprisingly, half of the Englishspeaking non-Latinos expressed concerns about psychotherapy based on past experiences- such as lack of rapport with a therapist, inability to have frequent enough visits, and, not learning enough coping skills for depression.

Language seemed to be a factor with combined treatment recommendation perceptions. Most all Spanish-speaking Latinos expressed negative feelings toward combined psychotherapy/pharmacotherapy depression treatment recommendations whereas most English speakers felt there was a benefit to the combined treatment. All providers supported psychotherapy and the majority supported pharmacotherapy and combined treatment recommendations.

\section{Theme 2: Receipt of Depression Treatment Recommendations}

Language also seemed to affect the receipt of combined depression treatment recommendations. Most English speakers reported receiving combined psychotherapy/pharmacotherapy depression treatment recommendations compared with only 2
Spanish speakers. Most PCPs reported that they recommend combined therapy to all patients based on the clinic guideline, regardless of language or ethnicity considerations.

\section{Theme 3: Barriers Impacting Treatment Recommendations}

Patients and PCPs reported unique barriers impacting uptake of depression treatment recommendations. Structural barriers such as transportation or work hours were reported as common barriers impacting treatment uptake for psychotherapy recommendations. Patients and providers also reported issues with access to timely appointments, appointment availability, frequency of psychotherapy visits, and shortage of therapists. Spanish speakers reported no availability of a Spanishspeaking therapist in the clinic, as confirmed by the provider's expressing the need for a bilingual and bicultural therapist.

Barriers impacting uptake of pharmacotherapy recommendations for English speakers include refill requests, issues with medication adjustments, and inability to reach the clinic for medication questions. Most Spanish speakers reported that they did not pick up their prescription from the pharmacy due to their negative perceptions of medications.

\section{Discussion}

This study provides information about patient language and receiving and accepting standard of care depression treatment recommendations (psychotherapy and pharmacotherapy). The data reveal 


\begin{tabular}{|c|c|}
\hline Theme/Subtheme/Frequency & Supporting Quotes \\
\hline \multicolumn{2}{|l|}{ Perceptions of treatment options } \\
\hline $\begin{array}{l}\text { Most Latinos (5 Spanish; } 4 \text { English) had negative feelings } \\
\text { about pharmacotherapy and preferred psychotherapy (4 } \\
\text { Spanish; } 5 \text { English), although } 4 \text { English-speaking Latinos } \\
\text { were willing to try depression medication }\end{array}$ & $\begin{array}{l}\text { "Me hace mucho daño la medicina y trato de evitarla lo más que } \\
\text { puedo. Translation: "Medicine does a lot of damage to me } \\
\text { and so I try to avoid it as much as I can." (Spanish-speaking, } \\
\text { White Latina) }\end{array}$ \\
\hline \multirow[t]{2}{*}{$\begin{array}{l}3 \text { English-speaking non-Latinos had negative experiences } \\
\text { with psychotherapy; } 4 \text { preferred pharmacotherapy }\end{array}$} & $\begin{array}{l}\text { "Counseling works better for me. I'm taking [depression] } \\
\text { medication right now but I don't know if it's working on } \\
\text { me...because I still cry a lot. I come out of counseling feeling } \\
\text { good." (English-speaking White Latina) }\end{array}$ \\
\hline & $\begin{array}{l}\text { "Medications have definitely helped because the times I run out } \\
\text { of refills, there's a noticeable difference. I've tried counseling } \\
\text { off and on but it hasn't helped." (English-speaking, White } \\
\text { non-Latino) }\end{array}$ \\
\hline \multicolumn{2}{|l|}{ Receipt of combined treatment recommendation } \\
\hline $\begin{array}{l}\text { Only } 2 \text { Spanish-speaking Latinos received combined } \\
\text { treatment recommendations; all } 6 \text { received psychotherapy } \\
\text { referrals }\end{array}$ & $\begin{array}{l}\text { "My doctor actually had someone go with me to behavioral } \\
\text { health to set up the appointment on the spot and asked how I } \\
\text { felt about depression medication. I said ok and he said he } \\
\text { would send a prescription to my pharmacy to try it out" } \\
\text { (English-speaking, White non-Latina) }\end{array}$ \\
\hline $\begin{array}{l}\text { Most English-speakers (5 Latinos; } 5 \text { non-Latinos) received } \\
\text { combined treatment recommendations. }\end{array}$ & $\begin{array}{l}\text { "He [doctor] asked about my medication and if I was following } \\
\text { through with behavioral health." (English-speaking White } \\
\text { non-Latino) }\end{array}$ \\
\hline \multicolumn{2}{|l|}{ Barriers impacting uptake of treatment recommendations } \\
\hline $\begin{array}{l}4 \text { Spanish-speaking Latinos stated that there were no } \\
\text { bilingual therapists available; English-speakers ( } 2 \text { Latinos; } 2 \\
\text { non-Latinos) stated that appointments were very limited }\end{array}$ & $\begin{array}{l}\text { "No podía con el consejero en esos días porque solo había una } \\
\text { persona disponible en inglés.Yo hablo ingles pero para sacar } \\
\text { tus sentimientos, para sacar todo lo que traes, necesitaba } \\
\text { hacerlo en español. Me dijeron que estaban buscando la ayuda } \\
\text { también en español. Translation: "I could not see the } \\
\text { counselor in those days because there was only one person } \\
\text { available in English. I speak English but to get your feelings } \\
\text { out, to get out everything you have, I needed to do it in } \\
\text { Spanish. They told me that they were looking for help in } \\
\text { Spanish as well." (Bilingual, Spanish-preferred White Latino) }\end{array}$ \\
\hline $\begin{array}{l}\text { English-speakers ( } 2 \text { Latinos; } 2 \text { non-Latinos) reported issues } \\
\text { with medication refills or requests for medication } \\
\text { adjustments }\end{array}$ & $\begin{array}{l}\text { "I called separately about Behavioral Health to see if I could get } \\
\text { a counselor and I was told that it was all the way backed up } \\
\text { until next year" (English-speaking, African-American non- } \\
\text { Latino) }\end{array}$ \\
\hline
\end{tabular}

Interviews conducted with 18 patients: 6 Spanish-speaking Latinos; 6 English-speaking Latinos; 6 English-speaking nonLatinos. Quotes include subgroup language, race, and gender-referring to females as Latina and males as Latino.

that language, which in this study is related to ethnicity, has an effect on depression treatment recommendations of PCPs. Being younger and an English speaker predicted whether the PCP recommended psychotherapy and pharmacotherapy to patients with depression. Older and Spanish-speaking patients were less likely to receive combined psychotherapy/pharmacotherapy treatment recommendations from their PCP. The sensitivity analysis found that ethnicity predicted whether the PCP recommended either psychotherapy or pharmacotherapy to depressed patients.

Qualitative interview analysis suggests that Spanish-speaking Latinos were less likely to receive combined psychotherapy/pharmacotherapy depression treatment recommendations. Latinos in this sample indicated they had negative perceptions about pharmacotherapy, and this is similar to other studies. ${ }^{19,20}$ US-born Latinos are less likely to follow mental health treatment plans and more likely to experience depression than other Latinos, and, than non-Latino whites. Further, other studies have found Latinos are fearful of mental health treatment leading to stigmatization and that the antidepressant medications are addictive; both perceptions lead to poor uptake of recommendations.

When combined therapy was recommended to Spanish-speaking Latinos, none followed through with the recommendation for medication and had difficulty accessing psychotherapy. Despite negative feelings about medications, English-speaking Latinos did follow through with psychotherapy/ 
pharmacotherapy recommendations for both medication and psychotherapy. Barriers impacting treatment recommendations aligned with themes from the literature: negative perception about medications, low patient follow-through with recommendations, and a shortage of behavioral health specialists as well as a shortage of behavioral health specialists who speak Spanish in an underserved community. ${ }^{8,11,12}$

\section{Study Limitations}

There are several limitations to this study, suggesting potential research opportunities in the future. This study is limited in that only 2 languages and 2 broad ethnic groups were examined. First, the use of purposive sampling of patients may have introduced selection bias. For example, men were considerably underrepresented in the study; of the 12 Latino patients in the qualitative interviews, only 2 were men. However, a random sample was not feasible given the purposive design to achieve the research aims. The findings from this study may only be generalizable to other underserved communities or community health centers. The study was designed to recruit patients as they screened positive for depression during their medical visit. Consequently, the study's design could have recruited patients who were being told they were depressed for the first time. Although the study attempted to add to the literature on depression in Latinos in a real-world setting, there were significant recruitment challenges for the qualitative phase of the study.

The ICSI guideline differs from many other guidelines in that combined treatment is recommended for all patients, even those with only mildto-moderate depression. However, the FQHC offered integrated behavioral health specialist services during medical visits making psychotherapy a feasible option for patients with depression. As a result, the practice's depression guideline indicates that the Integrated Behavioral Health Specialists would consult with the PCP at the visit and, if the patient had major depression, the patient would be referred for psychotherapy and receive a pharmacotherapy prescription. Thus, the current classification of patients who got combined therapy as standard of care and those referred for monotherapy, nonevidence based, or no treatment is consistent with the services and practices of the FQHC.

Cultural sensitivity issues presented unique challenges. The researchers were only successful with recruiting 1 Spanish-speaking Latino man. To mitigate the potential challenge of attrition, we offered the option of conducting interviews over the telephone. In addition, familism (focusing on familial values and wellbeing, as opposed to a focus on individual opportunities) was a key factor during recruitment. ${ }^{21}$ Often, family influenced participation in the study because they accompanied Latino patients to their medical appointments. From a PCP perspective, Familismo as a cultural asset, can sometimes feel cumbersome in a clinical or research context. But as a cultural protective factor among monolingual Spanish speakers and first generation Latinx it enhances rapport and compliance. ${ }^{21}$ Hence, it is important that researchers and PCPs alike find strategies to attend to this value when working with this population ${ }^{2-24}$ and more actively engage the families of their patients in discussions of health and treatment decisions.

Future studies should examine other minority groups and Spanish-speaking non-Latinos for comparisons. Given the few men scoring positive even on the PHQ-2, other studies should consider including men to get an overall understanding of possible gender differences in depression symptoms. One concern about the analysis in the present study is that being Spanish speaking may be a proxy variable for acculturation rather than simply a language preference. The medical chart did not include information about immigration status or acculturation and so language was used to examine a potential health disparity regarding access to and perceptions of mental health care. Finally, the PCPs expressed concern about whether the screening instrument (eg, PHQ-9) was being administered appropriately and consistently by the medical assistants in the clinic. At times, the patient did not indicate depressive symptoms on the scale but then expressed these symptoms to the PCP. Future research should address how these items should be assessed in a medical visit in an underserved, multicultural patient population.

\section{Conclusion}

Studying the Latino population as discrete subgroup does not account for the impact of language preference on attitudes toward mental illness, treatment recommendations, and overall reduction of depressive symptoms over time. Results of the present study indicate that being older or a Spanish-speaking Latino is related to fewer standard of care depression 
treatment recommendations by PCPs and potentially to a different uptake of PCP depression treatment recommendations by ethnicity. It is known that there is a shortage of mental health specialists across the nation and more so in underserved community health centers that serve Latino populations.

To see this article online, please go to: http://jabfm.org/content/ 32/6/904.full.

\section{References}

1. Holden K, McGregor B, Thandi P, et al. Toward culturally centered integrative care for addressing mental health disparities among ethnic minorities. Psychol Serv 2014;11:357-368.

2. Camacho Á, González P, Castañeda SF, et al. Improvement in depressive symptoms among Hispanic/Latinos receiving a culturally tailored IMPACT and problemsolving intervention in a community health center. Community Ment Health J 2015;51:385-392.

3. United States Department of Health \& Human Services, Health Resources and Services Administration. What are federally qualified health centers? Available from: https://www.hrsa.gov/opa/eligibilityandregistration/healthcenters/fqhc/index.html. Accessed April 13, 2017.

4. Bureau of Primary Health Care, Uniform Data System. Reporting Instructions for 2016 Center Data. Available from: http://www.bphcdata.net/docs/uds_ rep_instr.pdf. Accessed April 13, 2017.

5. Trangle M, Gursky J, Haight R, et al. Institute for Clinical Systems Improvement. Adult depression in primary care. Updated March 2016. Available from: https://www.icsi.org/guideline/depression.

6. United States Census Bureau. Hispanic Origin. Available from: https://www.census.gov/population/ hispanic/. Accessed March 23, 2016.

7. Reuland DS, Cherrington A, Watkins GS, Bradford DW, Blanco RA, Gaynes BN. Diagnostic accuracy of Spanish language depression-screening instruments. Ann Fam Med 2009;7:455-462.

8. Aponte-Rivera V, Dunlop BW, Ramirez C, et al. Enhancing Hispanic participation in mental health clinical research: development of a Spanish-Speaking depression research site. Depress Anxiety 2013;31:258-267.

9. Chang TE, Brill CD, Traeger L, et al. Association of race, ethnicity and language with participation in mental health research among adult patients in primary care. J Immigr Minor Health 2015;17:16601669.

10. Kalibatseva Z, Leong FT. A critical review of culturally sensitive treatments for depression: Recommendations for intervention and research. Psychol Serv 2014;11:433-450.

11. Kanter JW, Santiago-Rivera AL, Santos MM, et al.
A randomized hybrid efficacy and effectiveness trial of behavioral activation for Latinos with depression. Behav Ther 2015;46:177-192.

12. Thomas SB, Quinn SC, Butler J, Fryer CS, Garza MA. Toward a fourth generation of disparities research to achieve health equity. Annu Rev Public Health 2011;32:399-416.

13. Stephenson, J. Insights in depression screening at a Latino-serving, integrated FQHC clinic. Loma Linda University Electronic Theses, Dissertations \& Projects. 2017. Available from: https://scholarsrepository.llu.edu/etd/450.

14. Charmaz K, Belgrave LL. Qualitative interviewing and grounded theory analysis. The SAGE handbook of interview research: the complexity of the craft. Thousand Oaks, CA: SAGE Publications Inc.; 2012; 347-366.

15. Wulsin L, Somoza E, Heck J. The feasibility of using the Spanish PHQ-9 to screen for depression in primary care in Honduras. Prim Care Companion J Clin Psychiatry 2002;04:191-195.

16. Kroenke K, Spitzer RL, Williams JBW. The PHQ-9: validity of a brief depression severity measure. J Gen Intern Med 2001;16:606-613.

17. Huang FY, Chung H, Kroenke K, Delucchi KL, Spitzer RL. Using the Patient Health Questionnaire-9 to measure depression among racially and ethnically diverse primary care patients. J Gen Intern Med 2006;21:547-552.

18. Horevitz E, Organista KC, Arean PA. Depression treatment uptake in integrated primary care: how a "warm handoff" and other factors affect decision making by Latinos. Psychiatr Serv 2015;66:824-830.

19. Vargas SM, Cabassa LJ, Nicasio A, et al. Toward a cultural adaptation of pharmacotherapy: Latino views of depression and antidepressant therapy. Transcult Psychiatry 2015;52:244-273.

20. Lopez V, Sanchez K, Killian MO, Eghaneyan BH. Depression screening and education: an examination of mental health literacy and stigma in a sample of Hispanic women. BMC Public Health 2018;18:646-654.

21. Ayón C, Aisenberg E. Negotiating cultural values and expectations within the public child welfare system: A look at familismo and personalismo. Child Fam Social Work 2010;15:335-344.

22. Delgado M. Social work with Latinos: social, economic, political and cultural perspectives. Oxford, UK: Oxford University Press; 2017.

23. González-Prendes AA, Hindo C, Pardo Y. Cultural values integration in cognitive-behavioral therapy for a Latino with depression. Clin Case Studies 2011;10:376-394.

24. Williams LR, Ayón C, Marsiglia FF, Kiehne E, Ayers S. Acculturation profiles and associations with parenting among immigrant Latinos. Hispanic J Behav Sci 2017;39:452-469. 